\title{
Identificación de vacíos de investigación aplicada para restaurar ecosistemas terrestres en Colombia
}

\section{Identifying gaps of applied research to restore terrestrial ecosystems in Colombia}

\author{
Juan Garibello ${ }^{1 * \odot}$, Laura Riaño², Julián Cuellar², José Ignacio Barrera-Cataño3 \\ Wilson Ramírez ${ }^{1}$
}

Garibello, J., Riaño, L., Cuellar, J., Barrera-Cataño, J. I. y Ramírez, W. (2021). Identificación de vacíos de investigación aplicada para restaurar ecosistemas terrestres en Colombia. Colombia Forestal, 24(1), 88-107

Recepción: 7 de diciembre 2019

\section{Resumen}

Importantes desafíos en la restauración de ecosistemas terrestres colombianos pueden superarse de forma parcial a través de la investigación. Por tal razón, en el presente artículo de revisión hicimos un análisis bibliométrico de varios artículos para identificar los temas abordados y los vacíos usando un marco de trabajo publicado en 2017. Este marco se presenta como una agenda de investigación en ecología cuyo desarrollo tiene una aplicación directa en la práctica de la restauración. Encontramos 167 casos provenientes de 143 artículos; la mayoría trataron temas relacionados con planificación (35\%) y monitoreo de intervenciones $(25 \%)$. Pocos abordaron temas relacionados con implementación: obtención de propágulos (12\%), establecimiento de plántulas (13\%) y desempeño del material plantado (15\%). También, identificamos un sesgo geográfico, ya que $93 \%$ de los estudios se realizaron en las regiones Andina y Caribe; lo que equivale a menos de la mitad del territorio colombiano. Estos resultados y su análisis se presentan como una agenda preliminar de investigación en restauración que involucra intereses de diferentes actores.

Palabras clave: agenda de investigación para la restauración, costo-efectividad de la restauración, articulación ciencia-práctica.
Aprobación: 16 de septiembre 2020

\begin{abstract}
Important challenges faced in terrestrial ecosystems in Colombia might be partially overcome through research. For this reason, we made a bibliometric analysis with articles to identify addressed topics as well as gaps using a framework published in 2017 . This framework is presented as a research agenda based in ecology whose development has a direct application on restoration practice. We found 167 cases coming from 143 articles; the majority addressed topics related to planning (35\%) and monitoring of interventions (25\%). Few were related to implementation: sourcing propagules (12\%), seedling establishment (13\%) and performance of planted seedlings (15\%). We also identified a geographic bias since $93 \%$ of studies were made in the Andean and the Caribbean regions which are less than half of the national territory. These results and their analyses are presented as preliminary research agenda in restoration involving interests of different stakeholders.
\end{abstract}

Key words: cost-effectiveness of restoration, research agenda for restoration, science-practice articulation.

\footnotetext{
Instituto de Investigación de Recursos Biológicos Alexander von Humboldt. Bogotá, Colombia.

Universidad Distrital Francisco José de Caldas. Bogotá, Colombia.

3 Pontificia Universidad Javeriana, Bogotá, Colombia.

jcgaribellop@gmail.com. Autor para correspondencia.
} 


\section{INTRODUCCIÓN}

Son aproximadamente 12 millones de personas en Colombia las que habitan en zonas expuestas a desabastecimiento hídrico como resultado de la pérdida y degradación de ecosistemas naturales (Instituto de Hidrología, Meteorología y Estudios Ambientales, 2010). Así mismo, cerca del $21 \%$ del territorio nacional requiere restauración ecológica de manera prioritaria debido a sus niveles de deterioro y a que se trata de las zonas con mayor densidad poblacional (Ramírez et al., 2016). La tarea de revertir esta situación se ha asumido en parte a través de la restauración ecológica y enfoques afines (rehabilitación, recuperación). Estas estrategias de conservación están ampliamente respaldadas por la normatividad y políticas estatales. Por ejemplo, la restauración está incluida en el artículo 80 de la Constitución Política vigente (Aguilar-Garavito et al., 2017) y algunas entidades territoriales como la Secretaría de Ambiente de Bogotá han ejecutado planes de manera continua desde el año 2000 (Murcia et al., 2017).

Pese al respaldo normativo y a la ejecución continua de programas, el progreso e impacto de la restauración distan de ser óptimos en Colombia. El desarrollo de esta práctica presenta carencias importantes en aspectos como gobernanza participativa, la planificación y el monitoreo (Murcia et al., 2016). Además, la percepción entre agentes del gremio es que las metas gubernamentales nunca se han cumplido y que las plantaciones vinculadas a iniciativas precedentes no persisten en la actualidad (Murcia et al., 2017a). La promoción y desarrollo de investigaciones ha sido identificada como uno de los ámbitos de trabajo para mejorar esta situación tanto en el orden nacional (Murcia et al., 2017b) cómo en el regional (Holl, 2017). Esta concepción estaría asociada, al menos, con dos circunstancias. Por un lado, la insuficiencia de la información disponible para administrar la incertidumbre inherente de un proceso de largo plazo como la restauración ecológica (Murcia et al., 2017a). De otra parte, un contexto de complejidad creciente que apunta a la implementación de iniciativas a gran escala (Chazdon et al., 2017) en un contexto de emergencia climática (Harris et al., 2006).

La investigación asociada a la restauración contribuye al logro de estándares técnicos que soporten los proyectos (Burbidge et al., 2011; Menz et al., 2013); además, posibilita la elaboración de conceptos claros, modelos y metodologías (Society for Ecological Restoration, 2004); y hace un aporte sustancial a la identificación de estrategias de intervención costo-efectivas (Holl, 2017). No obstante, con alguna frecuencia uno de los campos de conocimiento asociado (la ecología de la restauración) no responde a este desafío al no abordar aspectos prácticos (Cabin et al., 2010) o al no hacer explícita la utilidad práctica de los hallazgos. Esto ocurre debido a la ausencia de un marco que defina el tipo de investigación requerida, cuáles son las preguntas asociadas y cómo estos dos se relacionan con las distintas fases de la práctica de la restauración (MiIler et al., 2017). A nivel nacional, a la ausencia de un vínculo claro entre ciencia y práctica, se añade la falta de una agenda que optimice el uso de los recursos limitados para investigación. No existen ejercicios que evalúen el aporte de la ecología de la restauración a la restauración ecológica en el marco de la practicidad; es decir, en el que los resultados de investigación tengan una aplicación inmediata o que incidan en la efectividad de las iniciativas de restauración. Sin embargo, es de señalar que Murcia y Guariguata (2014) analizaron la evolución, retos y oportunidades de la restauración en Colombia; pero, a diferencia del presente estudio, su trabajo no se basó en producción científica publicada sino en proyectos.

Por lo ya dicho, se presenta el siguiente estudio; asumiendo que importantes desafíos de la restauración ecológica pueden afrontarse mediante investigación y que parte de esta debe tener una aplicabilidad explícita. El objetivo es, entonces, identificar vacíos de conocimiento científico cuyo 
abordaje contribuya a mejorar la práctica de la restauración de ecosistemas terrestres. Para tal fin se utilizó el marco de trabajo propuesto por Miller et al. (2017), el cual tiene como propósito acortar la brecha entre ciencia y práctica mediante el desarrollo de una agenda de investigación. Una de las ventajas de este marco es que las preguntas que conforman la agenda se dividen en fases que son asimilables a las etapas de un proyecto de restauración. El desarrollo de dicho marco apunta a la conformación de ecosistemas autosostenibles, resilientes y biodiversos. Por ello, este estudio es una primera evaluación del estado de la ecología de la restauración en Colombia y de su aporte a aspectos fundamentales de su faceta práctica: la restauración ecológica.

\section{MATERIALES Y MÉTODOS}

Se utilizaron las bases de datos Google Académico y Scopus para buscar artículos científicos publicados en revistas que estuviesen indexadas en Scimago o en Publindex en el momento de la búsqueda. Para tal fin, se utilizaron los binomios "restauración ecológica" AND "Colombia" junto con "ecología de la restauración" AND "Colombia". También se incluyeron binomios en los cuales se reemplazó "restauración" con los términos afines "rehabilitación" y "recuperación. La búsqueda se hizo tanto en español cómo en inglés entre abril y junio de 2019. Inicialmente, aparecieron aproximadamente 17000 resultados, de los cuales se seleccionaron solamente artículos que incluyeran "restauración" o los términos mencionados, bien fuera en el título, resumen o palabras clave. No se tuvieron en cuenta tesis de grado, guías, protocolos o documentos de trabajo. Los criterios finales de inclusión fueron que el artículo abordara una pregunta o problema de investigación claramente definido y que fuera clasificable dentro del marco de trabajo adoptado. Esto último limitó la búsqueda a investigaciones en ecosistemas terrestres o anfibios.
Las publicaciones fueron clasificadas según la propuesta de Miller et al. (2017). Esta propuesta surge como resultado de la poca efectividad de algunos programas de restauración, debida a su vez a la poca correspondencia entre las necesidades de la práctica de la restauración y la ecología de la restauración. Adicional a esto, seleccionamos esta propuesta o marco de trabajo pues incluye aspectos de la mayor importancia pero que probablemente están ausentes de la mayoría de proyectos en Colombia. Por ejemplo: el aprovechamiento de la regeneración natural y de la siembra como estrategias de restauración, la diversidad genética, la consideración del compartimiento suelo, el papel del área intervenida en la conectividad y la evaluación de la resiliencia del área restaurada.

La propuesta presenta 36 preguntas de investigación agrupadas en cinco fases: I) definición de objetivos y planificación para la restauración; II) obtención de propágulos; III) optimización del establecimiento de plántulas; IV) facilitación del crecimiento y sobrevivencia de plántulas en campo; y $V$ ) resiliencia y sostenibilidad del ecosistema en restauración y su integración con el resto del paisaje. A diferencia de la propuesta original, en la fase I no se incluyeron solamente ecosistemas de referencia, sino también ecosistemas degradados. Además, se transformaron las preguntas en temas para hacer menos restrictiva la clasificación de las publicaciones que encontramos.

Para identificar vacíos en términos de cobertura geográfica, las publicaciones se clasificaron de acuerdo con su región natural y ecosistema. Debido a que los autores no utilizan un único sistema para denominar el ecosistema en el cual se desarrolló la investigación (o a veces no lo mencionan), fue necesario utilizar la altitud y los datos climáticos reportados, así como referencias de apoyo (por ejemplo: Cleef et al., 1984 y Pizano y García, 2014). Se utilizó un análisis $\chi^{2}$ de bondad de ajuste para establecer si las fases se han abordado con la misma frecuencia y para establecer si en cuanto a número de estudios las regiones tenían similar representatividad. Con el mismo análisis se estableció si la 
proporcionalidad entre fases era similar al clasificar las publicaciones por ecosistemas y por regiones. Dado que el análisis exige una distribución normal de las proporciones, fue necesario agrupar las categorías que tuvieron un menor número de casos. De este modo, se comparó el bosque subhúmedo y húmedo de cordillera contra un grupo conformado por todos los demás ecosistemas; igualmente, se comparó la región Andina con un grupo conformado por todas las demás regiones.

\section{RESULTADOS}

Encontramos un total de 143 estudios publicados a partir de 1996; siendo la mayoría (79 \%) publicados a partir de 2010 (figura 1; anexo 1). Dado que 21 artículos abordaron dos o más temas, el análisis se hizo sobre 167 casos. Las fases tratadas con mayor frecuencia fueron la "Definición de objetivos y planificación para la restauración", junto con "Resiliencia, sostenibilidad e integración del ecosistema en restauración", con 59 y 42 casos, respectivamente. Las otras tres fases: "Obtención de propágulos", "Establecimiento de plántulas a partir de semillas" y
"Crecimiento y sobrevivencia de plántulas en campo", se abordaron con menor frecuencia, en 20, 22 y 23 casos, respectivamente (figura 2). "Establecimiento de plántulas a partir de semillas" fue la fase que se trató con mayor frecuencia en aquellos artículos que exploraron más de un tema (10 estudios). "Almacenamiento de semillas" y "Condiciones físicas, químicas e hidrológicas del sustrato y su manipulación" fueron los temas más recurrentes con los que se combinó "Establecimiento" en este tipo de estudios.

Para definir objetivos y planificar la restauración, la mayoría de casos trataron sobre la caracterización de ecosistemas tanto de referencia como degradados. En segundo lugar, se identificaron especies de plantas con potencial para la restauración mientras que unos pocos casos abordaron la dinámica sucesional e identificación de ecosistemas de referencia, respectivamente. Dentro de los casos que registraron el impacto de la restauración en la resiliencia y sostenibilidad del ecosistema, la mayoría midieron procesos ecosistémicos y reportaron la recuperación de fauna y recursos asociados,; mientras que un número considerablemente menor se centró en el potencial de regeneración (tabla 1).

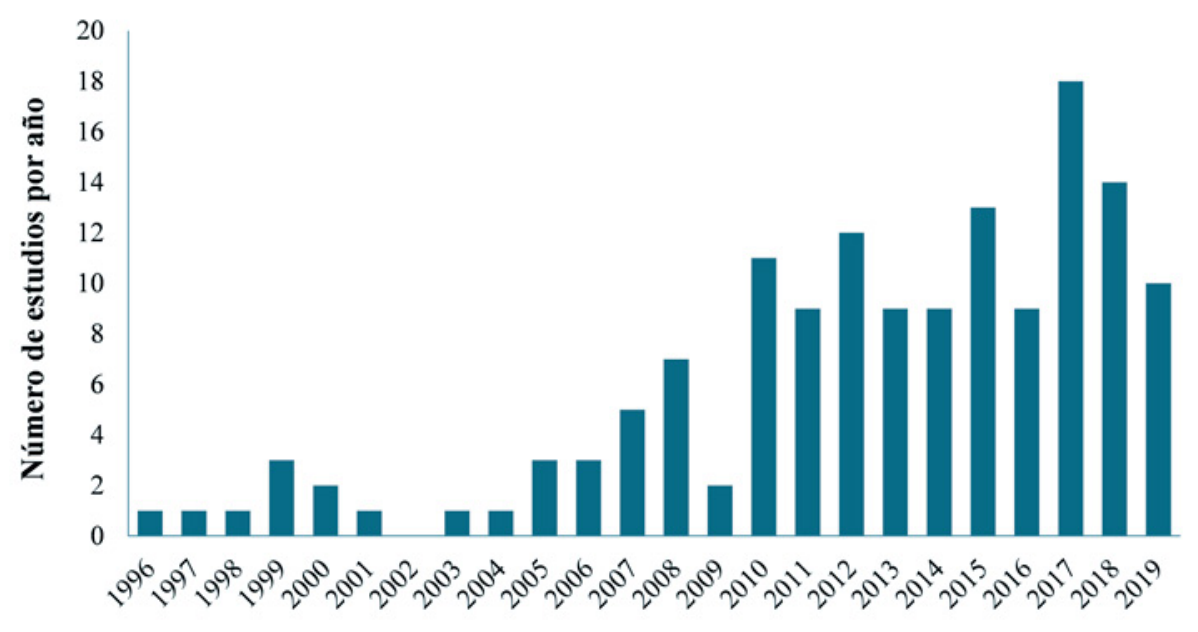

Figura 1. Distribución del número de estudios publicados que abordan aspectos prácticos de ecología de la restauración en Colombia (total estudios $n=143$ ). 


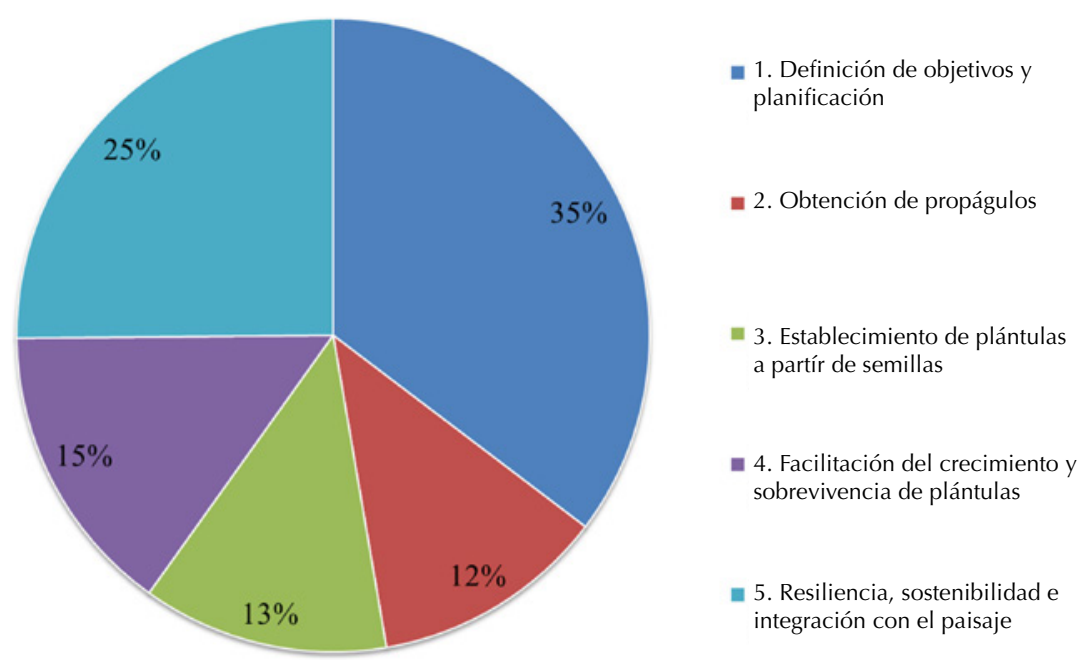

Figura 2. Distribución porcentual del número de estudios en fases de investigación práctica en ecología de la restauración en Colombia publicados entre 1996 y junio de 2019 (n=143 artículos, 21 de estos abarcan más de una fase o tema para un total de 167 casos).

Tabla 1. Fases y temas de importancia práctica abordados en artículos de ecología de la restauración en Colombia publicados hasta junio de 2019

\begin{tabular}{|c|c|c|}
\hline Fase & Tema abordado & $\begin{array}{c}\text { Número de } \\
\text { estudios }\end{array}$ \\
\hline $\begin{array}{l}\text { Definición de objetivos } \\
\text { y planificación para la } \\
\text { restauración. }\end{array}$ & $\begin{array}{c}\text { Caracterización de ecosistemas de referencia o degradados. } \\
\text { Especies con uso potencial para la restauración. } \\
\text { Dinámica sucesional. } \\
\text { Identificación de ecosistemas de referencia. }\end{array}$ & $\begin{array}{c}33 \\
14 \\
8 \\
4\end{array}$ \\
\hline $\begin{array}{l}\text { Obtención de } \\
\text { propágulos. }\end{array}$ & $\begin{array}{c}\text { Genética. } \\
\text { Bancos de semillas. } \\
\text { Contribución de la regeneración natural y a la dispersión. } \\
\text { Almacenamiento de semillas. } \\
\text { Oferta espacio-temporal de semillas. } \\
\text { Mejora del aporte de la regeneración natural y de la dispersión. }\end{array}$ & $\begin{array}{l}5 \\
4 \\
1 \\
3 \\
3 \\
4\end{array}$ \\
\hline $\begin{array}{l}\text { Optimización del estable- } \\
\text { cimiento de plántulas. }\end{array}$ & $\begin{array}{c}\text { Manejo de condiciones ambientales para inducir germinación sin considerar condi- } \\
\text { ción interna de la semilla en términos fisiológicos, genéticos o anatómicos. } \\
\text { Tratamiento a plántulas o estacas antes de plantar. } \\
\text { Momento de siembra y manipulación del sitio para optimizar germinación } \\
\text { y establecimiento. }\end{array}$ & $\begin{array}{l}12 \\
5 \\
4\end{array}$ \\
\hline $\begin{array}{l}\text { Facilitación del } \\
\text { crecimiento y } \\
\text { sobrevivencia de } \\
\text { plántulas en campo. }\end{array}$ & $\begin{array}{l}\text { Condiciones físicas, químicas e hidrológicas del sustrato y su manipulación. } \\
\text { Condiciones biofísicas por encima del suelo y su manipulación } \\
\text { Incidencia y uso de biota edáfica. } \\
\text { Amenazas a la restauración y su manejo o mitigación. } \\
\text { Patrones de plantación o siembra para optimizar desempeño de plántulas. }\end{array}$ & $\begin{array}{l}9 \\
6 \\
5 \\
3 \\
2\end{array}$ \\
\hline $\begin{array}{l}\text { Resiliencia, sostenibilidad e } \\
\text { integración del ecosistema } \\
\text { en restauración con el resto } \\
\text { del paisaje. }\end{array}$ & $\begin{array}{l}\text { Procesos ecosistémicos. } \\
\text { Recuperación de fauna, hábitats y recursos asociados. } \\
\text { Potencial de regeneración. }\end{array}$ & $\begin{array}{l}20 \\
17 \\
5\end{array}$ \\
\hline
\end{tabular}


La proporción de publicaciones es similar para genética de poblaciones, bancos de semillas y mejoras al aporte de la regeneración y la dispersión dentro de la fase de obtención de propágulos. El almacenamiento y oferta espacio-temporal de semillas, junto con la evaluación de la regeneración y la dispersión, se abordaron en menor proporción. Germinación controlando factores ambientales es el tema predominante para la fase de optimización del establecimiento de plántulas. Dentro de esta fase también aparecen, pero en menor frecuencia, el tratamiento de las plántulas o estacas antes de plantar y manipulación del sitio de siembra para optimizar la germinación y el establecimiento. La evaluación y manipulación del sustrato es el tema más recurrente dentro de las publicaciones que exploraron la facilitación del desempeño de plántulas en campo. Otras publicaciones relacionadas con este tema se centraron en las condiciones microclimáticas (por ejemplo: competencia por luz y clima), así como incidencia y uso de biota edáfica, amenazas a la restauración y patrones de siembra y plantación (tabla 1).

Casi la mitad de temas propuestos por Miller et al. (2017), dentro de su marco para investigar aspectos prácticos de la restauración, no se han abordado en las publicaciones sobre restauración de ecosistemas terrestres colombianos (tabla 2). En cuanto a la obtención de propágulos, no se encontraron estudios sobre el uso de horizontes orgánicos de suelo y sus técnicas de almacenaje y transferencia. Tampoco hay publicaciones acerca de mejoramiento de producción de semillas en poblaciones naturales, producción de semillas ex situ ni técnicas de propagación asexual. No hay publicaciones sobre técnicas para superar dormancia orgánica (Baskin y Baskin, 2014) ni publicaciones sobre técnicas de siembra como parte de la optimización en el establecimiento de plántulas. Tampoco se han publicado trabajos que asocien la modificación de la topografía o la modificación de la superficie del suelo con el crecimiento y sobrevivencia de material plantado. Así mismo, aún no se publican estudios que evalúen la resiliencia en ecosistemas en restauración después de disturbios (o bajo estrés ambiental) ni se han publicado trabajos sobre el papel de polinizadores en la producción y viabilidad de semillas en estos sitios. Otro aspecto sobre el que no encontramos publicaciones es el posible impacto o el impacto real de un sitio en restauración en la matriz paisajística circundante (tabla 2).

Como ocurrió con las fases y temas propuestos por Miller et al. (2017), la cobertura geográfica de las publicaciones sobre ecología de la restauración en Colombia revela sesgos. El $73 \%$ de los estudios fue desarrollado en la región Andina; más de la mitad de ellos se realizó en bosques de cordillera sub-húmedos y húmedos mientras que los restantes fueron en bosque seco, páramo, bosque húmedo tropical, xerofítico andino y ronda de humedal. Los dos últimos en altitud superior a 2250. El Caribe es la segunda región con más publicaciones, pero con un porcentaje muy inferior al de la Andina; 22 estudios equivalentes al $15 \%$ del total, la mayoría de ellos en bosque seco y en manglar. Los otros corresponden a dunas costeras y bosque húmedo tropical. El agregado para la Amazonia, la Orinoquía y la región Pacífico corresponde tan solo a 10 publicaciones (7 \%): para bosque húmedo tropical, altillanura y manglar. Cuatro de los 143 estudios se hicieron tanto en la región Andina así como en la Caribe al analizar la diversidad genética de poblaciones distantes de plantas en el bosque seco (tabla 3 ).

El análisis $\chi^{2}$ de bondad de ajuste confirmó que las fases propuestas por el marco de trabajo adoptado no se han abordado con la misma frecuencia $\left(\chi^{2}=34.5 ; p<0.0001 ;\right.$ g.I. $\left.=4\right)$ y que existe un desbalance marcado en la frecuencia con la que se han hecho estudios en las diferentes regiones $\left(\chi^{2}\right.$ $=298.82 ; p<0.0001 ;$ g.I. $=4)$. La proporcionalidad en que se han abordado las cinco fases en los bosques subhúmedos y húmedos de cordillera es similar a la proporcionalidad con la que se han abordado en los otros ecosistemas $\left(\chi^{2}=6.44\right.$; $p=0.1684 ;$ g.l. $=4)$. Por el contrario, la frecuencia con la que se han abordado en la región Andina 
Tabla 2. Temas de importancia práctica no abordados por estudios sobre ecología de la restauración en Colombia publicados hasta junio de 2019

\begin{tabular}{|c|c|}
\hline Fase & Tema no abordado \\
\hline $\begin{array}{l}\text { Definición de objetivos } \\
\text { y planificación para la } \\
\text { restauración. }\end{array}$ & Ninguno. \\
\hline Obtención de propágulos. & $\begin{array}{c}\text { Efecto del origen de los propágulos para minimizar impactos negativos a la restauración. } \\
\text { Mezclar genotipos de diferentes sitios (para incrementar potencial evolutivo) versus } \\
\text { utilizar genotipos locales (para maximizar adaptación). } \\
\text { Incidencia de la endogamia o la baja diversidad genética de la población de origen } \\
\text { en el éxito de la restauración. } \\
\text { Incidencia de la mezcla de genotipos de poblaciones diferentes en el éxito de la restauración. } \\
\text { Optimización del uso de horizontes orgánicos de suelo considerando su cosecha, } \\
\text { almacenaje y transferencia. } \\
\text { Técnicas para incrementar la producción de semillas viables en poblaciones naturales. } \\
\text { Productividad en cultivos para producir semillas versus diversidad genética y viabilidad } \\
\text { de las semillas. }\end{array}$ \\
\hline $\begin{array}{l}\text { Optimización del estable- } \\
\text { cimiento de plántulas. }\end{array}$ & $\begin{array}{c}\text { Técnicas para acortar período de dormancia orgánica evaluando condición fisiológica, } \\
\text { bioquímica, genética y anátomica de las semillas. } \\
\text { Técnicas de siembra (incluyendo mecanización y recubrimientos de semillas). } \\
\text { Genética. }\end{array}$ \\
\hline $\begin{array}{l}\text { Facilitación del creci- } \\
\text { miento y sobrevivencia de } \\
\text { plántulas en campo. }\end{array}$ & $\begin{array}{c}\text { Modificaciónes de la topografía. } \\
\text { Modificación de la superficie (a través de arado, acolchados cultivos para cubrir el suelo, etc.) } \\
\text { Genética. }\end{array}$ \\
\hline $\begin{array}{l}\text { Resiliencia, sostenibilidad } \\
\text { e integración del ecosiste- } \\
\text { ma en restauración con el } \\
\text { resto del paisaje. }\end{array}$ & $\begin{array}{c}\text { Resiliencia a disturbios y estrés ambiental en sitios en restauración. } \\
\text { Efecto del tamaño de las poblaciones y su diversidad genética sobre la producción } \\
\text { y viabilidad de las semillas. } \\
\text { Manejo de polinizadores para mejorar la producción y viabilidad de semillas en áreas } \\
\text { en restauración. } \\
\text { Localización, orientación y tamaño del sitio a restaurar para mejorar conectividad, integración } \\
\text { con el paisaje circundante e influencia de la restauración a escala de paisaje. }\end{array}$ \\
\hline
\end{tabular}

difiere ligeramente de la frecuencia con la que se han abordado en las otras regiones $\left(\chi^{2}=9.58 ; p=\right.$ $0.0481 ;$ g.I. $=4)$ (tabla 3). El porcentaje de estudios de establecimiento de plántulas en la región Andina es casi el doble del porcentaje para las otras regiones ( $15 \%$ vs. $8 \%$ ). En las otras regiones es un poco más alto el porcentaje con el que se ha abordado la evaluación de ecosistemas en restauración comparado con la Andina (29\% versus $24 \%$ ).

\section{DISCUSIÓN}

Los análisis de la literatura científica (como el que acá se presentó) son importantes para entender la amplitud o el alcance de un campo científico como la ecología de la restauración (Borgman y Furner, 2002; Song y Zhao, 2013). Los resultados obtenidos identifican los aspectos que se han abordado con mayor o menor frecuencia a nivel 
Tabla 3. Estudios de ecología de la restauración en regiones y ecosistemas de Colombia publicados hasta junio de 2019

\begin{tabular}{|c|c|c|}
\hline Región & Ecosistema & $\begin{array}{c}\text { Número } \\
\text { de estudios }\end{array}$ \\
\hline Amazonas & Bosque húmedo tropical. & 3 \\
\hline \multirow[t]{7}{*}{ Andina } & $\begin{array}{l}\text { Bosque de cordillera subhúme- } \\
\text { do-húmedo. }\end{array}$ & 71 \\
\hline & Bosque seco tropical. & 14 \\
\hline & Páramo. & 10 \\
\hline & Bosque húmedo tropical. & 7 \\
\hline & Xerofítico andino. & 2 \\
\hline & Ronda humedal. & 1 \\
\hline & Indeterminado. & 2 \\
\hline $\begin{array}{l}\text { Andina/ } \\
\text { Caribe }\end{array}$ & Bosque seco tropical. & 4 \\
\hline \multirow[t]{4}{*}{ Caribe } & Bosque seco tropical. & 10 \\
\hline & Manglar. & 9 \\
\hline & Dunas. & 1 \\
\hline & Bosque húmedo tropical. & 2 \\
\hline Orinoquía & Altillanura. & 2 \\
\hline \multirow[t]{2}{*}{ Pacífico } & Bosque húmedo tropical. & 4 \\
\hline & Manglar. & 1 \\
\hline
\end{tabular}

nacional e identifican aquellos que todavía no se han explorado. También sugieren que la investigación asociada a restauración ecológica en Colombia es insuficiente si se considera la extensión de la degradación y la megadiversidad involucrada (Etter et al., 2008). Existe, además, un sesgo marcado en las fases y temas abordados, así como en las regiones y ecosistemas estudiados. El $60 \%$ de los casos revisados corresponden solamente a dos de las cinco fases del marco de trabajo adoptado. Así mismo, solo cuatro temas de un total de 39 que propone el marco adoptado (incluyendo genética), tienen presencia en el $51 \%$ del total de casos. La región Andina concentra el $73 \%$ de publicaciones. El número de publicaciones (143) y los sesgos temáticos y geográficos sugieren, entonces, que la información científica local con la que cuentan las iniciativas de restauración en Colombia es aún escasa.

La producción científica nacional en restauración ecológica o en ecología de la restauración es comparable a la de otros países latinoamericanos como Argentina (105 artículos entre 1996 y 2013, según Rovere, 2013) y México (160 artículos entre 1995 y 2016, según López-Barrera et al., 2017). En contraste, la producción es ostensiblemente inferior a la de los tres primeros países con mayor número de publicaciones: Estados Unidos (1248), China (819) y Australia (339) (Romanelli et al., 2018). Las diferencias entre estos países y los países latinoamericanos son proporcionales al porcentaje del PIB invertido en investigación (Banco Mundial, 2019). Esta brecha ratifica que una de las alternativas de financiación que se debe fortalecer para que haya más investigación en Colombia es el establecimiento de alianzas entre autoridades ambientales, academia, ONG y empresas privadas; tal como se ha hecho en el marco de algunas compensaciones ambientales por construcción de infraestructura y explotación minera. Sin embargo, es necesario mencionar que uno de los desafíos frecuentes en este escenario de gestión es la asignación de suficientes recursos y tiempo para investigación y monitoreo. De otra parte, durante la pesquisa se constató la existencia de aproximadamente 100 tesis de pregrado y posgrado, lo cual sugiere que existe trabajo y potencial para incrementar significativamente el número de publicaciones.

El $60 \%$ de los casos que evaluamos aborda la definición de objetivos de la restauración y la medición de su éxito. Al revisar los temas que conforman estas dos fases del marco de trabajo, es evidente que estos trabajos tienen un énfasis más descriptivo que experimental; contrario a las tres fases que han sido menos exploradas: la obtención de propágulos, el establecimiento de plántulas y la facilitación del desempeño de estas plántulas. Si bien el tratamiento de temas predominante incluye aspectos esenciales como la caracterización de ecosistemas (Perkins y Leffler, 2018) y el monitoreo 
de indicadores de escala pequeña (Aguilar-Garavito y Ramírez, 2015), creemos que la investigación en restauración debe adoptar con mayor frecuencia un enfoque basado en experimentos. Esta decisión implica la comparación metódica de tratamientos y tecnologías que conduzca a la identificación de estrategias costo-efectivas cuya aplicación contribuiría a su vez a superar grandes retos; entre otros, la escasez de recursos financieros, la necesidad de ampliar significativamente la cobertura espacial de los proyectos y el ajuste de políticas gubernamentales identificados por Murcia et al. (2016).

Una de las fases menos estudiadas según nuestro análisis fue la obtención de propágulos. En la consecución de costo-efectividad, un tema de la mayor relevancia dentro de esta fase es la restauración pasiva ("Contribución de la regeneración natural y la dispersión" y "Mejora del aporte de la regeneración natural y de la dispersión" en el marco adoptado). No obstante, solo en 10 casos se trató dicho tema, incluyendo aquellos de la fase cinco agrupados bajo el título "Potencial de regeneración". Es necesario que se tipifique a través de investigación dónde es viable esta estrategia, al considerar variables como historia de uso y cercanía de vegetación remanente (Chazdon, 2014), junto con abundancia de árboles dentro del sitio a restaurar (Esquivel et al., 2008). Una vez se establezca que existe una oferta de propágulos tanto in situ (Williams-Linera et al., 2016), así como vía dispersión (Robinson y Handel, 2000), es necesario investigar sobre el uso de estrategias complementarias entre sí; como, por ejemplo, la erradicación sucesiva de plantas invasoras (Gaertner et al., 2012; Kettenring y Reinhardt-Adams, 2011) y el uso de perchas tanto naturales como artificiales (Reid y Holl, 2013).

En tan solo 20 casos (13\%) se exploró el establecimiento de plántulas. Si bien la siembra de semillas es menos efectiva que plantación en términos de número de plántulas sobrevivientes, sus costos más bajos y la consecución de resultados más rápidos hacen que sea una opción a tener en cuenta (Masarei et al., 2019), así sea como estrategia complementaria a la plantación (Ceccon et al., 2015). Dentro de esta fase del marco de trabajo se propone como prioritario el abordaje de los temas "Momento de siembra y manipulación del sitio junto" con "Técnicas de siembra", pues implican la superación de barreras que están presentes en la mayoría de escenarios de restauración de ecosistemas terrestres. Dichas barreras son: desecación de la semilla, pudrición, depredación y competencia con vegetación pre-existente (Reid y Holl, 2013). La adopción de siembra supone conseguir suficientes semillas que sean viables, que estén sanas, que sean diversas genéticamente y que no se vea afectada la población de origen como resultado de la colecta. Estos requisitos reiteran que se necesita más investigación en los temas de la fase obtención de propágulos.

La publicación de investigaciones sobre el crecimiento y sobrevivencia de plántulas en campo también ha sido escasa (15\%, es decir, 24 casos). Este déficit llama la atención, considerando que la principal estrategia de restauración a nivel nacional es la plantación de árboles. Los aspectos o temas que propone Miller et al. (2017) en esta fase incluyen entender y manejar el suelo a partir de sus características físicas, químicas y biológicas. Así como también el manejo del suelo concebido como sustrato, en el que es necesario explorar aspectos como arado, acolchados, herbáceas no agresivas que compitan con gramíneas invasoras y modificación de la topografía. La investigación que vincula el suelo y el desempeño de material plantado es aún más apremiante si se tienen en cuenta hallazgos recientes en el ámbito local, los cuales sugieren que el abordaje de suelos en proyectos de restauración en Colombia presenta falencias conceptuales y técnicas que amenazan el éxito de esta práctica (Durán-Dueñas, 2018). Otro componente importante de esta fase sobre desempeño de plántulas (que no se menciona pero que está implícito) es la interacción entre los individuos plantados, así como entre estos y la vegetación preexistente. Dicha interacción se presenta tanto a nivel subterráneo como aéreo (Cahill, 1999), en concordancia con los temas de la fase. Una manera de abordar 
este componente sería la experimentación con los patrones espaciales y composicionales de las plantaciones o siembras, junto con experimentación en el manejo de la vegetación preexistente.

En las publicaciones encontradas, el componente genético no se ha tratado más allá de la necesaria caracterización de poblaciones de plantas con potencial para la restauración. De acuerdo con el marco adoptado y con autores como Kettenring et al. (2014), es necesario establecer el efecto de la diversidad genética en el éxito de la restauración, no solo en el largo plazo sino también en etapas intermedias del proceso (como el establecimiento de plántulas, su crecimiento y sobrevivencia). Seguir esta recomendación es muy importante pues no siempre la diversidad genética está relacionada con indicadores de corto o mediano plazo como el desempeño del material plantado o la formación de toda una cobertura vegetal. Se puede conformar un dosel con una o dos especies, cada una proveniente de una fuente semillera única; pero esto afectaría la sostenibilidad de la restauración en el largo plazo (Thomas et al., 2014), en relación con temas como cambio climático o viabilidad poblacional.

Aunque la fase cinco del marco de trabajo fue la segunda con más casos (41, de un total de 167), presenta vacíos que requieren investigación. Según el análisis realizado no se han hecho estudios para establecer si los ecosistemas en restauración son resilientes a disturbios o a estrés ambiental. La manipulación de disturbios sería poco probable en sitios en restauración, ya sea por restricciones legales o porque no se pueden controlar los agentes de disturbio. En este contexto, documentar científicamente los disturbios, sus efectos y la respuesta ecológica dependería tanto de la selección y medición oportuna de los indicadores así como de la aplicación de varios tratamientos que puedan tener una respuesta disímil frente a situaciones no siempre controlables como presencia de ganado, incendios, especies invasoras, entre otras. El mismo razonamiento valdría para reportar los efectos de episodios asociados con cambio climático.
Otro apartado de esta fase sobre el cual tampoco se ha publicado investigación es el efecto de la restauración a escala de paisaje o los efectos del paisaje sobre la restauración. Para tal fin es necesario considerar variables como el tamaño, la ubicación y el aporte a la conectividad del sitio en restauración; entendiendo que dichas variables a su vez inciden en procesos bióticos y abióticos que determinan la sostenibilidad ecosistémica de la intervención (Menz et al., 2013). Estos factores inciden, además, en una escala mayor a la del sitio en restauración en cuanto a funciones como flujos hidrológicos, dinámicas metapoblacionales, rango de acción de especies animales (Shoo y Caterall, 2013) y, en general, en los servicios ecosistémicos.

El análisis no solo muestra tendencias en los temas abordados, sino que también revela profundos sesgos en la distribución geográfica de las investigaciones. El 94 \% fueron realizadas en las regiones Andina y Caribe, lo cual coincide parcialmente con la ejecución de proyectos (Murcia y Guariguata, 2014). Si bien estas dos regiones presentan las mayores necesidades de restauración en términos de superficie degradada (Ramírez et al., 2016), también es cierto que la tasa y la severidad de la degradación durante los últimos años en las regiones restantes requiere mayor atención por parte de los investigadores. El avance de la minería y explotación maderera en el Pacífico (Valois-Cuesta, 2016; Meyer et al., 2019), de la deforestación en la Amazonía (Murad y Pearse, 2018) y el avance de la agroindustria en la Orinoquía (Cárdenas-Aguilera, 2018) hacen imperativa la aplicación inmediata de un marco investigativo como el que presentamos en este artículo.

Junto con los vacíos de investigación detectados mediante este análisis bibliométrico, se destacan otros aspectos relativos al progreso de la ciencia y la práctica de la restauración de acuerdo con la literatura (Holl, 2010 y Holl, 2017). El avance de la restauración no depende exclusivamente del abordaje de temas prioritarios como los considerados en este trabajo, sino que, además, obedece a la forma como se aborden dichos temas. 
Idealmente, los proyectos tanto de investigación como de intervención deberían tener una cobertura espacial mayor que incluya suficiente heterogeneidad ambiental para llegar a conclusiones más contundentes. Así mismo, deben generar resultados que tengan importancia para diferentes audiencias: comunidades y autoridades locales, academia local y externa y quienes formulan las políticas. También es indispensable involucrar los diferentes actores en todas las etapas del proceso. Las recomendaciones desde la literatura también incluyen un período de seguimiento prolongado tanto para proyectos como para investigaciones. En tal sentido, se destaca que el rango de tiempo abarcado por los estudios que analizamos es lo suficientemente amplio como para asegurar que dicho requerimiento ha sido tenido en cuenta por quienes han publicado. El rango es de 0.75 a 80 años $(\bar{x}=19.3 ; \sigma=17.6)$.

Aunque el alcance del análisis realizado cubre solamente aspectos ecológicos, resulta fundamental armar e implementar una agenda de investigación que cubra aspectos económicos y sociales. No se trata solamente de reportar con mucha mayor frecuencia los costos y los beneficios económicos asociados a la restauración, tal como se ha hecho con sistemas silvopastoriles (Murgueitio et al., 2011 y Calle et al., 2013) y con plantaciones forestales no nativas (Brancalion et al., 2019), sino también de establecer la contribución de la restauración a la generación de empleo, el PIB, la seguridad alimentaria, almacenamiento de carbono y la mitigación de la pobreza (Ding et al., 2017). Así mismo, resulta prioritario evaluar la incidencia de la participación comunitaria en las diferentes etapas de los procesos; incluyendo monitoreo y creación de línea base a partir de ciencia ciudadana.

Finalmente, es necesario buscar estrategias de divulgación complementarias a la publicación de investigaciones en revistas científicas indexadas, dado que no todos los actores tienen acceso a este tipo de información. Por tal motivo, es necesario divulgar y discutir los resultados a través de medios como talleres, programas de extensión y redes sociales (Matzek et al., 2014). Posiblemente, la interacción frecuente y dialogante entre los diferentes actores sea preferible a la generación de manuales y guías debido a que lo primero puede ser más efectivo al enfrentar los desafíos únicos de cada proyecto en lo social, lo político y lo ecológico. Por esta misma razón, se enfatiza en que el conocimiento a compartir debe ser de origen predominantemente local, emulando iniciativas que han integrado exitosamente ciencia, práctica y diversidad de actores como el Pacto de la Mata Atlántica en Brasil (Melo et al., 2013) y el Programa Gondwana Link en el suroeste de Australia (Bradby et al., 2016).

\section{CONCLUSIÓN}

Las investigaciones que versan sobre la ecología de la restauración, analizadas desde un marco de practicidad, han contribuido al desarrollo de la restauración ecológica en Colombia. No obstante, esta contribución resulta insuficiente al tener en cuenta la magnitud de la degradación, la cantidad de publicaciones y los sesgos temáticos y geográficos. En cuanto a los temas, las publicaciones han abordado principalmente la caracterización de ecosistemas, así como el registro y análisis de algunos indicadores de éxito a pequeña escala.

Si bien se trata de aspectos esenciales en la práctica de la restauración, también es necesario explorar temáticas de investigación en las cuales se optimice la obtención de propágulos y el uso de estrategias como la siembra de semillas y la plantación. Esto requiere que se adopte de manera más frecuente (pero no exclusiva) un enfoque experimental, el cual se diferencie del enfoque más descriptivo que ha predominado a la fecha. En este contexto, el abordar temas como diversidad genética, técnicas de siembra, relación suelo-planta, interacción planta-planta y función de la restauración a escala de paisaje aportaría una contribución significativa al diseño de estrategias más costo-efectivas, tanto en el corto como en el 
largo plazo. La implementación y monitoreo de esas estrategias aportaría, a su vez, a la superación de grandes desafíos; entre los que están la poca disponibilidad de recursos económicos, la necesidad de mayor cobertura espacial de los proyectos y el ajuste de políticas.

Por otro lado, se ha investigado con mucha mayor frecuencia en las regiones Andinas y Caribe, por lo que se identifica que ello va en consecuencia con el grado de transformación de dichas regiones. Sin embargo, la tasa de deterioro actual y la severidad de las alteraciones hacen necesario que se haga más investigación en las regiones de la Amazonia, Orinoquia y Pacífico, teniendo como base el marco a partir del cual se desarrolló el presente trabajo.

Los resultados obtenidos y la consideración del marco de trabajo utilizado como soporte son un paso importante para definir una agenda de investigación aplicada cuyo desarrollo informe sobre cómo restaurar ecosistemas más diversos, sostenibles y resilientes. Del mismo modo, también apuntan a ser un punto de encuentro entre la academia y otros actores, en el que unos y otros puedan identificar cuáles son los insumos que se necesitan desde la ciencia para la correcta ejecución de cada una de las etapas de un proceso de restauración.

\section{AGRADECIMIENTOS}

A Tatiana Sanjuan por sus comentarios sobre la estructura del manuscrito.

\section{CONFLICTO DE INTERESES}

Los autores declaran no tener conflicto de intereses

\section{CONTRIBUCIÓN POR AUTOR}

J.G. concibió la investigación, hizo los análisis definitivos y redactó el manuscrito. L.R. y J.C. colectaron la información e hicieron los análisis preliminares. J.I.B.C. y W.R. revisaron y retroalimentaron versiones previas de este texto.

\section{REFERENCIAS}

Aguilar-Garavito, M. y Ramírez, W. (eds.) (2015). Monitoreo a procesos de restauración ecológica aplicado a ecosistemas terrestres. Instituto de Investigación de Recursos Biológicos Alexander von Humboldt.

Aguilar-Garavito, M., Schlesinger, S., Ramírez, W., Hernández, A. y Franco, A. (2017). La restauración ecológica. Una mirada política y normativa. En L. A. Moreno, G. I. Andrade y L. F. Ruíz-Contreras (eds.), Biodiversidad 2016. Estado y tendencias de la biodiversidad continental de Colombia (pp. 6869.). Instituto de Investigación de Recursos Biológicos Alexander von Humboldt.

Banco Mundial (2019). Research and Development Expenditure (percentage of gross domestic product). https://data.worldbank.org/indicator/gb.xpd.rsdv. gd.zs?name_desc=false

Baskin, C. C. y Baskin, J. M. (2014). Seeds: ecology, biogeography, and, evolution of dormancy and germination. Elsevier.

Borgman, C. L. y Furner, J. (2002). Scholarly communication and bibliometrics. Annual Review of Information Science and Technology, 36(1), 2-72.

Bradby, K., Keesing, A. y Wardell-Johnson, G. (2016). Gondwana Link: connecting people, landscapes, and livelihoods across southwestern Australia. Restoration Ecology, 24(6), 827-835. https://doi.org/10.1111/rec.12407

Brancalion, P. H., Amazonas, N. T., Chazdon, R. L., van Melis, J., Rodrigues, R. R., Silva, C. C., Sorrini, T.B. y Holl, K. D. (2019). Exotic eucalypts: from demonized trees to allies of tropical forest restoration? Journal of Applied Ecology. https://doi.org/10.1111/1365-2664.13513

Burbidge, A. H., Maron, M., Clarke, M. F., Baker, J., Oliver, D. L. y Ford, G. (2011). Linking science and practice in ecological research and 
management: how can we do it better? Ecological Management \& Restoration, 12(1), 54-60. https://doi.org/10.1111/j.1442-8903.2011.00569.x

Cabin, R. J., Clewell, A., Ingram, M., McDonald, T. y Temperton, V. (2010). Bridging restoration science and practice: results and analysis of a survey from the 2009 society for ecological restoration international meeting. Restoration Ecology, 18(6), 783-788. https://doi.org/10.1111/j.1526-100X.2010.00743.x

Cahill Jr., J. F. (1999). Fertilization effects on interactions between above-and belowground competition in an old field. Ecology, 80(2), 466-480. https://doi.org/10.1890/0012-9658(1999)080[046 6:FEOIBA]2.0.CO;2

Calle, Z., Murgueitio, E., Chará, J., Molina, C. H., Zuluaga, A. F. y Calle, A. (2013). A strategy for scaling-up intensive silvopastoral systems in Colombia. Journal of Sustainable Forestry, 32(7), 677-693. https://doi.org/10.1080/10549811.2013.817338

Cárdenas-Aguilera, J. F. (2018). Aproximación a la producción agroindustrial en la Orinoquía: el caso de la soya (Glycine max) en el municipio de Puerto López-Meta (tesis maestría). Pontificia Universidad Javeriana, Bogotá, Colombia. http://hdl.handle.net/10554/34348

Ceccon, E., González, E. J. y Martorell, C. (2016). Is direct seeding a biologically viable strategy for restoring forest ecosystems? Evidences from a Meta-analysis. Land Degradation \& Development, 27(3), 511-520. https://doi.org/10.1002/ldr.2421

Chazdon, R. L. (2014). Second growth: the promise of tropical forest regeneration in an age of deforestation. University of Chicago Press.

Chazdon, R. L., Brancalion, P. H., Lamb, D., Laestadius, L., Calmon, M. y Kumar, C. (2017). A policy-driven knowledge agenda for global forest and landscape restoration. Conservation Letters, 10(1), 125-132. https://doi.org/10.1111/conl.12220

Cleef, A. M., Rangel, O., van der Hammen, T. y Jaramillo, R. (1984). La vegetación de las selvas del transecto Buritaca. Studies on tropical Andean ecosystems, 2, 267-407.

Ding, H., Faruqi. S., Wu, A., Altamirano, J., Anchondo, O., Verdone, M., Zamora, R., Chazdon, R. y
Vergara, W. (2017). Roots of prosperity. The economics and finance of restoring land. World Resources Institute.

Durán-Dueñas,J.C.(2018)AnálisisAmbientaldelSueloen Proyectos de Restauración Ecológica de Ecosistemas Terrestres en Colombia (2003-2016) (tesis de maestría). Universidad Nacional de Colombia, Bogotá. https://repositorio.unal.edu.co/handle/unal/68827

Esquivel, M. J., Harvey, C. A., Finegan, B., Casanoves, F. y Skarpe, C. (2008). Effects of pasture management on the natural regeneration of neotropical trees. Journal of Applied Ecology, 45(1), 371-380. https://doi.org/10.1111/j.1365-2664.2007.01411.x

Etter, A., McAlpine, C. y Possingham, H. (2008). Historical patterns and drivers of landscape change in Colombia since 1500: a regionalized spatial approach. Annals of the Association of American Geographers, 98(1), 2-23.

Gaertner, M., Fisher, J., Sharma, G. y Esler, K. (2012). Insights into invasion and restoration ecology: time to collaborate towards a holistic approach to tackle biological invasions. NeoBiota, 12, 57.

Giardina, C. P., Litton, C. M., Thaxton, J. M., Cordell, S., Hadway, L. J. y Sandquist, D. R. (2007). Science driven restoration: a candle in a demon haunted world-response to Cabin (2007). Restoration Ecology, 15(2), 171-176. https://doi.org/10.1111/j.1526-100X.2007.00227.x

Harris, J. A., Hobbs, R. J., Higgs, E. y Aronson, J. (2006). Ecological restoration and global climate change. Restoration Ecology, 14(2), 170-176. https://doi.org/10.1111/j.1526-100X.2006.00136.x

Holl, K. D. (2010). Writing for an international audience. Restoration Ecology, 18(2), 135-137. https://doi.org/10.1111/j.1526-100X.2009.00646.x

Holl, K. D. (2017). Research Directions in Tropical Forest Restoration. Annals of the Missouri Botanical Garden, 102(2), 237-251. https://doi.org/10.3417/2016036

Instituto de Hidrología, Meteorología y Estudios Ambientales (2010). Estudio nacional del agua 2010. Instituto de Hidrología, Meteorología y Estudios Ambientales. 
Kettenring, K. M. y Reinhardt-Adams, C. (2011). Lessons learned from invasive plant control experiments: a systematic review and meta-analysis. Journal of Applied Ecology, 48(4), 970-979. https://doi.org/10.1111/j.1365-2664.2011.01979.x

Kettenring, K. M., Mercer, K. L., Reinhardt-Adams, C. y Hines, J. (2014). Application of genetic diversity-ecosystem function research to ecological restoration. Journal of Applied Ecology, 51(2), 339-348. https://doi.org/10.1111/1365-2664.12202

López-Barrera, F., Martínez-Garza, C. y Ceccon, E. (2017). Ecología de la restauración en México: estado actual y perspectivas. Revista Mexicana de Biodiversidad, 88, 97-112. https://doi.org/10.1016/j.rmb.2017.10.001

Masarei, M., Guzzomi, A. L., Merritt, D. J. y Erickson, T. E. (2019). Factoring Restoration Practitioner Perceptions into Future Design of Mechanical Direct Seeders for Native Seeds. Restoration Ecology. https://doi.org/10.1111/rec.13001

Matzek, V., Covino, J., Funk, J. L. y Saunders, M. (2014). Closing the knowing-doinggapin invasiveplantmanagement: accessibility and interdisciplinarity of scientific research. Conservation Letters, 7(3), 208-215. https://doi.org/10.1111/conl.12042

Melo, F. P., Pinto, S. R., Brancalion, P. H., Castro, P. S., Rodrigues, R. R., Aronson, J. y Tabarelli, M. (2013). Priority setting for scaling-up tropical forest restoration projects: Early lessons from the Atlantic Forest Restoration Pact. Environmental Science \& Policy, 33, 395-404. https://doi.org/10.1016/j.envsci.2013.07.013

Menz, M. H., Dixon, K. W. y Hobbs, R. J. (2013). Hurdles and opportunities for landscape-scale restoration. Science, 339(6119), 526-527. https://doi.org/10.1126/science.1228334

Meyer, V., Saatchi, S., Ferraz, A., Xu, L., Duque, A., García, M. y Chave, J. (2019). Forest degradation and biomass loss along the Chocó region of Colombia. Carbon Balance and Management, 14(1), 2.

Miller, B. P., Sinclair, E. A., Menz, M. H., Elliott, C. P., Bunn, E., Commander, L. E., Dalziel, E., David, E., Davis, B., Erikcson, T. D., Golos, P. J., Krauss, S. L., Lewandrowski, W., Ellery-Mayence,
C., Merino-Martín, L., Merritt, D. J., Nevill, P.G., Phillips, R. D., Ritchie, A. L., Ruoss, S. y Stevens, J. C. (2017). A framework for the practical science necessary to restore sustainable, resilient, and biodiverse ecosystems. Restoration Ecology, 25(4), 605-617.

https://doi.org/10.1111/rec.12475

Murad, C. A. y Pearse, J. (2018). Landsat study of deforestation in the Amazon region of Colombia: Departments of Caquetá and Putumayo. Remote Sensing Applications:Society andEnvironment, 11, 161-171. https://doi.org/10.1016/j.rsase.2018.07.003

Murcia, C. y Guariguata, M. R. (2014). La restauración ecológica en Colombia: tendencias, necesidades y oportunidades. Documentos ocasionales 107. Centro para la Investigación Forestal Internacional.

Murcia, C., Guariguata, M. R., Andrade, Á., Andrade, G. I., Aronson, J., Escobar, E. M., Etter, A., Moreno, F. H., Ramírez, W. y Montes, E. (2016). Challenges and prospects for scaling-up ecological restoration to meet international commitments: Colombia as a case study. Conservation Letters, 9(3), 213-220. https://doi.org/10.1111/conl.12199

Murcia, C., Guariguata, M. R., Peralvo, M. y Gálmez, V. (2017a). La restauración de bosques andinos tropicales: avances, desafíos y perspectivas del futuro. Documentos Ocasionales 170. https://doi.org/10.17528/cifor/006524

Murcia, C., Guariguata, M. R., Quintero-Vallejo, E. y Ramírez, W. (2017b). La restauración ecológica en el marco de las compensaciones por pérdida de biodiversidad en Colombia: Un análisis crítico. Vol. 176. Centro para la Investigación Forestal Internacional.

Murgueitio, E., Calle, Z., Uribe, F., Calle, A. y Solorio, B. (2011). Native trees and shrubs for the productive rehabilitation of tropical cattle ranching lands. Forest Ecology and Management, 261(10), 1654-1663. https://doi.org/10.1016/j.foreco.2010.09.027

Perkins, L. B. y Leffler, A. J. (2018). Conceptualizing ecological restoration: a concise and adaptable framework for researchers and practitioners. Restoration Ecology, 26(6), 1024-1028. https://doi.org/10.1111/rec.12881 
Pizano, C. y García, H. (eds.) (2014). El bosque seco tropical en Colombia. Instituto de Investigación de Recursos Biológicos Alexander von Humboldt.

Ramírez, W., Murcia, C., Guariguata, M., Thomas, E., Aguilar, M. y Isaacs, P. (2016). Restauración ecológica. Los retos para Colombia. En M. F Gómez, L. A. Moreno, G. I. Andrade y C. Rueda (eds.), Biodiversidad 2015. Estado y tendencias de la biodiversidad continental de Colombia (pp. 72-73). Instituto de Investigación de Recursos Biológicos Alexander von Humboldt.

Reid, J. L. y Holl, K. D. (2013). Arrival $\neq$ survival. Restoration Ecology, 21(2), 153-155. https://doi.org/10.1111/j.1526-100X.2012.00922.x

Robinson, G.R. y Handel, S.N. (2000). Directing spatial patterns of recruitment during an experimental urban woodland reclamation. Ecological Applications, 10, 174-188. https://doi.org/10.1890/1051-0761(2000)010[01 74:DSPORD]2.0.CO;2

Romanelli, J. P., Fujimoto, J. T., Ferreira, M. D. y Milanez, D. H. (2018). Assessing ecological restoration as a research topic using bibliometric indicators. Ecological Engineering, 120, 311-320. https://doi.org/10.1016/j.ecoleng.2018.06.015

Rovere, A. E. (2015). Review of the science and practice of restoration in Argentina: increasing awareness of the discipline. Restoration Ecology, 23(5), 508-512. https://doi.org/10.1111/rec.12240
Shoo, L. P. y Catterall, C. P. (2013). Stimulating natural regeneration of tropical forest on degraded land: approaches, outcomes, and information gaps. Restoration Ecology, 21(6), 670-677. https://doi.org/10.1111/rec.12048

Society for Ecological Restoration (2004). The SER international primer on ecological restoration. Society for Ecological Restoration.

Song, Y. y Zhao, T. (2013). A bibliometric analysis of global forest ecology research during 2002-2011. SpringerPlus, 2(1), 204.

Thomas, E., Jalonen, R., Loo, J., Boshier, D., GaIlo, L., Cavers, S., Bordács, S., Smith, P. y Bozzano, M. (2014). Genetic considerations in ecosystem restoration using native tree species. Forest Ecology and Management, 333, 66-75. https://doi.org/10.1016/j.foreco.2014.07.015

Valois-Cuesta, H. (2016). Sucesión primaria y ecología de la revegetación de selvas degradadas por minería en el Chocó, Colombia: bases para su restauración ecológica (tesis de doctorado). Instituto Universitario de Investigación en Gestión Forestal Sostenible, Universidad de Valladolid, España. https://doi.org/10.35376/10324/19089

Williams-Linera, G., Bonilla-Moheno, M. y López-Barrera, F. (2016). Tropical cloud forest recovery: the role of seed banks in pastures dominated by an exotic grass. New Forests, 47(3), 481-496. https://doi.org/10.1007/s11056-016-9526-8 
Anexo 1. Base de datos de casos evaluados

\begin{tabular}{|c|c|c|c|}
\hline Año & Primer autor & Revista & Sección según Miller et al. (2017) \\
\hline 1996 & Argüello & Agronomía Colombiana & Resiliencia, sostenibilidad e integración con el paisaje \\
\hline 1997 & Murcia & Forest Ecology and Management & Resiliencia, sostenibilidad e integración con el paisaje \\
\hline 1998 & Arens & American Fern Society & Definición de objetivos y planificación \\
\hline 1999 & Elster & Hybrobiologa & Establecimiento de plántulas a partir de semillas \\
\hline 1999 & Elster & Hybrobiologa & $\begin{array}{c}\text { Facilitación del crecimiento y sobrevivencia } \\
\text { de plántulas }\end{array}$ \\
\hline 1999 & Aide & Restoration Ecology & Establecimiento de plántulas a partir de semillas \\
\hline 1999 & Aide & Restoration Ecology & $\begin{array}{c}\text { Facilitación del crecimiento y sobrevivencia } \\
\text { de plántulas }\end{array}$ \\
\hline 1999 & Perdomo & Marine Pollution Bulletin & Obtención de propágulos \\
\hline 2000 & Posada & Restoration Ecology & Definición de objetivos y planificación \\
\hline 2000 & Posada & Restoration Ecology & Establecimiento de plántulas a partir de semillas \\
\hline 2000 & Elster & Forest Ecology and Management & $\begin{array}{l}\text { Facilitación del crecimiento y sobrevivencia } \\
\text { de plántulas }\end{array}$ \\
\hline 2001 & Díaz & Colombia Forestal & Definición de objetivos y planificación \\
\hline 2003 & Cortés & Caldasia & Definición de objetivos y planificación \\
\hline 2004 & Cardona & Colombia Forestal & Obtención de propágulos \\
\hline 2005 & Romero & Colombia Forestal & Definición de objetivos y planificación \\
\hline 2005 & Lentijo & Ornitología Colombiana & Resiliencia, sostenibilidad e integración con el paisaje \\
\hline 2005 & Groenendijk & Plant Ecology & Definición de objetivos y planificación \\
\hline 2006 & Kattan & Restoration Ecology & Resiliencia, sostenibilidad e integración con el paisaje \\
\hline 2006 & Benavides & Journal of Tropical Ecology & Definición de objetivos y planificación \\
\hline 2006 & Galindo & Acta Biológica Colombiana & $\begin{array}{l}\text { Facilitación del crecimiento y sobrevivencia } \\
\text { de plántulas }\end{array}$ \\
\hline 2007 & Castañeda & Colombia Forestal & Establecimiento de plántulas a partir de semillas \\
\hline 2007 & Castañeda & Colombia Forestal & Establecimiento de plántulas a partir de semillas \\
\hline 2007 & Guacaneme & Universitas Scientiarum & Resiliencia, sostenibilidad e integración con el paisaje \\
\hline 2007 & Ochoa & Universitas Scientiarum & $\begin{array}{c}\text { Facilitación del crecimiento y sobrevivencia } \\
\text { de plántulas }\end{array}$ \\
\hline 2007 & Roncancio & $\begin{array}{l}\text { Boletín Científico del Centro } \\
\text { de Museos }\end{array}$ & Resiliencia, sostenibilidad e integración con el paisaje \\
\hline 2007 & Arbeláez & Hybrobiologa & Obtención de propágulos \\
\hline 2008 & Montenegro & Revista de Biología Tropical & Definición de objetivos y planificación \\
\hline 2008 & Cantillo & Colombia Forestal & Definición de objetivos y planificación \\
\hline 2008 & Cantillo & Colombia Forestal & Obtención de propágulos \\
\hline 2008 & Cantillo & Colombia Forestal & Obtención de propágulos \\
\hline 2008 & Castaño & $\begin{array}{l}\text { Revista Facultad Nacional de } \\
\text { Agronomía }\end{array}$ & Resiliencia, sostenibilidad e integración con el paisaje \\
\hline 2008 & Gruezmacher & Colombia Forestal & $\begin{array}{c}\text { Facilitación del crecimiento y sobrevivencia } \\
\text { de plántulas }\end{array}$ \\
\hline 2008 & Montenegro & Revista de Biología Tropical & Definición de objetivos y planificación \\
\hline 2008 & Pedraza & Zootecnía Tropical & Resiliencia, sostenibilidad e integración con el paisaje \\
\hline 2008 & Arellano & Caldasia & Definición de objetivos y planificación \\
\hline 2009 & Cantillo & Colombia Forestal & Definición de objetivos y planificación \\
\hline 2009 & Montero & $\begin{array}{c}\text { Boletín Científico del Centro } \\
\text { de Museos }\end{array}$ & Definición de objetivos y planificación \\
\hline 2010 & Yepes & Revista Lasallista de Investigación & Resiliencia, sostenibilidad e integración con el paisaje \\
\hline
\end{tabular}




\begin{tabular}{|c|c|c|c|}
\hline 2010 & Yepes & Revista de Biología Tropical & Definición de objetivos y planificación \\
\hline 2010 & Castellanos & Acta Biológica Colombiana & Resiliencia, sostenibilidad e integración con el paisaje \\
\hline 2010 & González & Colombia Forestal & Obtención de propágulos \\
\hline 2010 & Giraldo & $\begin{array}{l}\text { Boletín Científico del Centro } \\
\text { de Museos }\end{array}$ & Obtención de propágulos \\
\hline 2010 & Rivera & Avances Investigación en Ingeniería & $\begin{array}{l}\text { Facilitación del crecimiento y sobrevivencia } \\
\text { de plántulas }\end{array}$ \\
\hline 2010 & Medina & Colombia Forestal & Definición de objetivos y planificación \\
\hline 2010 & Kattan & Tropical Conservation Science & Resiliencia, sostenibilidad e integración con el paisaje \\
\hline 2010 & Ascuntar & $\begin{array}{l}\text { Boletín Científico del Centro } \\
\text { de Museos }\end{array}$ & Resiliencia, sostenibilidad e integración con el paisaje \\
\hline 2010 & García & Restoration Ecology & Resiliencia, sostenibilidad e integración con el paisaje \\
\hline 2010 & Barliza & Acta Biológica Colombiana & Resiliencia, sostenibilidad e integración con el paisaje \\
\hline 2011 & Castro & Acta Biológica Colombiana & Definición de objetivos y planificación \\
\hline 2011 & Fernández & $\begin{array}{c}\text { Boletín Científico del Centro } \\
\text { de Museos }\end{array}$ & Resiliencia, sostenibilidad e integración con el paisaje \\
\hline 2011 & Bohórquez & $\begin{array}{l}\text { Boletín Científico del Centro } \\
\text { de Museos }\end{array}$ & Definición de objetivos y planificación \\
\hline 2011 & Pérez & Colombia Forestal & Establecimiento de plántulas a partir de semillas \\
\hline 2011 & Duarte & Tecciencia & Definición de objetivos y planificación \\
\hline 2011 & Giraldo & Insect Conservation and Diversity & Resiliencia, sostenibilidad e integración con el paisaje \\
\hline 2011 & Cortés & Biodiversity and Conservation & Definición de objetivos y planificación \\
\hline 2011 & Domínguez & Restoration Ecology & Resiliencia, sostenibilidad e integración con el paisaje \\
\hline 2011 & Domínguez & Restoration Ecology & Resiliencia, sostenibilidad e integración con el paisaje \\
\hline 2011 & Del Valle & Revista de Biología Tropical & Definición de objetivos y planificación \\
\hline 2012 & Camargo & Acta Agronómica & Definición de objetivos y planificación \\
\hline 2012 & Solorza & Luna Azul & $\begin{array}{l}\text { Facilitación del crecimiento y sobrevivencia } \\
\text { de plántulas }\end{array}$ \\
\hline 2012 & Chaves & $\begin{array}{l}\text { Revista de Investigación Agraria } \\
\text { y Ambiental }\end{array}$ & Definición de objetivos y planificación \\
\hline 2012 & Arcila & Biota Colombiana & Definición de objetivos y planificación \\
\hline 2012 & Hurtado & Caldasia & Establecimiento de plántulas a partir de semillas \\
\hline 2012 & Méndez & Revista Colombiana de Entomología & Resiliencia, sostenibilidad e integración con el paisaje \\
\hline 2012 & Henao & Restoration Ecology & Obtención de propágulos \\
\hline 2012 & Restrepo & Revista de Biología Tropical & Definición de objetivos y planificación \\
\hline 2012 & Mosquera & Geoderma & Resiliencia, sostenibilidad e integración con el paisaje \\
\hline 2012 & Blanco & Revista de Biología Tropical & Definición de objetivos y planificación \\
\hline 2012 & Ruiz & Revista Gestión y Ambiente & Definición de objetivos y planificación \\
\hline 2012 & Melo & Colombia Forestal & $\begin{array}{l}\text { Facilitación del crecimiento y sobrevivencia } \\
\text { de plántulas }\end{array}$ \\
\hline 2013 & Bohórquez & Colombia Forestal & Definición de objetivos y planificación \\
\hline 2013 & Rojas & Revista de Biología Tropical & Establecimiento de plántulas a partir de semillas \\
\hline 2013 & Gómez & Ecological Engineering & $\begin{array}{l}\text { Facilitación del crecimiento y sobrevivencia } \\
\text { de plántulas }\end{array}$ \\
\hline 2013 & Flórez & Revista de Biología Tropical & Resiliencia, sostenibilidad e integración con el paisaje \\
\hline 2013 & Betancourt & $\begin{array}{l}\text { Boletín de Investigación Marina } \\
\text { Costera }\end{array}$ & Definición de objetivos y planificación \\
\hline 2013 & Rubiano & $\begin{array}{c}\text { Boletín Científico del Centro } \\
\text { de Museos }\end{array}$ & Resiliencia, sostenibilidad e integración con el paisaje \\
\hline 2013 & Peña & Forest Ecology and Management & Resiliencia, sostenibilidad e integración con el paisaje \\
\hline 2013 & Calle & Restoration Ecology & Resiliencia, sostenibilidad e integración con el paisaje \\
\hline
\end{tabular}




\begin{tabular}{|c|c|c|c|}
\hline 2013 & Calle & Restoration Ecology & Resiliencia, sostenibilidad e integración con el paisaje \\
\hline 2013 & Muñoz & $\begin{array}{l}\text { Agriculture Ecosystems } \\
\text { and Environment }\end{array}$ & Definición de objetivos y planificación \\
\hline 2014 & Beltrán & Biota Colombiana & $\begin{array}{c}\text { Facilitación del crecimiento y sobrevivencia } \\
\text { de plántulas }\end{array}$ \\
\hline 2014 & Hernández & Biota Colombiana & $\begin{array}{l}\text { Facilitación del crecimiento y sobrevivencia } \\
\text { de plántulas }\end{array}$ \\
\hline 2014 & Acero & $\begin{array}{c}\text { Revista de la Academia Colombiana } \\
\text { de Ciencias }\end{array}$ & Definición de objetivos y planificación \\
\hline 2014 & Acero & $\begin{array}{c}\text { Revista de la Academia Colombiana } \\
\text { de Ciencias }\end{array}$ & Establecimiento de plántulas a partir de semillas \\
\hline 2014 & Avella & Biota Colombiana & Definición de objetivos y planificación \\
\hline 2014 & García & $\begin{array}{c}\text { Revista de Investigación Agraria } \\
\text { y Ambiental }\end{array}$ & Definición de objetivos y planificación \\
\hline 2014 & Ramírez & Plant and Soil & Resiliencia, sostenibilidad e integración con el paisaje \\
\hline 2014 & Castro & Caldasia & Definición de objetivos y planificación \\
\hline 2014 & Konnerup & Estuarine, Costal and Shelf Science & Resiliencia, sostenibilidad e integración con el paisaje \\
\hline 2014 & Sepúlveda & Acta Biológica Colombiana & Establecimiento de plántulas a partir de semillas \\
\hline 2015 & Vargas & Revista de Biología Tropical & Establecimiento de plántulas a partir de semillas \\
\hline 2015 & Vargas & Revista de Biología Tropical & Obtención de propágulos \\
\hline 2015 & Posada & Revista EIA & Definición de objetivos y planificación \\
\hline 2015 & Collantes & $\begin{array}{c}\text { Revista de Investigación Agraria } \\
\text { y Ambiental }\end{array}$ & Definición de objetivos y planificación \\
\hline 2015 & Collantes & $\begin{array}{c}\text { Revista de Investigación Agraria } \\
\text { y Ambiental }\end{array}$ & Definición de objetivos y planificación \\
\hline 2015 & Moreno & Acta Biológica Colombiana & Establecimiento de plántulas a partir de semillas \\
\hline 2015 & Moreno & Acta Biológica Colombiana & $\begin{array}{c}\text { Facilitación del crecimiento y sobrevivencia } \\
\text { de plántulas }\end{array}$ \\
\hline 2015 & Moreno & Acta Biológica Colombiana & $\begin{array}{l}\text { Facilitación del crecimiento y sobrevivencia } \\
\text { de plántulas }\end{array}$ \\
\hline 2015 & Moreno & Acta Biológica Colombiana & $\begin{array}{l}\text { Facilitación del crecimiento y sobrevivencia } \\
\text { de plántulas }\end{array}$ \\
\hline 2015 & Bocanegra & $\begin{array}{l}\text { Boletín Científico del Centro } \\
\text { de Museos }\end{array}$ & Definición de objetivos y planificación \\
\hline 2015 & Bocanegra & $\begin{array}{l}\text { Boletín Científico del Centro } \\
\text { de Museos }\end{array}$ & Definición de objetivos y planificación \\
\hline 2015 & López & Luna Azul & Definición de objetivos y planificación \\
\hline 2015 & Gutierrez & Colombia Forestal & Establecimiento de plántulas a partir de semillas \\
\hline 2015 & Ávila & Acta Biológica Colombiana & Establecimiento de plántulas a partir de semillas \\
\hline 2015 & Ávila & Acta Biológica Colombiana & $\begin{array}{l}\text { Facilitación del crecimiento y sobrevivencia } \\
\text { de plántulas }\end{array}$ \\
\hline 2015 & Alvarado & Acta Biológica Colombiana & Definición de objetivos y planificación \\
\hline 2015 & Lizarazo & Acta Biológica Colombiana & $\begin{array}{l}\text { Facilitación del crecimiento y sobrevivencia } \\
\text { de plántulas }\end{array}$ \\
\hline 2015 & Basto & Universitas Scientiarum & Establecimiento de plántulas a partir de semillas \\
\hline 2015 & Herrera & Environmental Entomology & Resiliencia, sostenibilidad e integración con el paisaje \\
\hline 2015 & Sierra & $\begin{array}{l}\text { Revista Facultad Nacional } \\
\text { de Agronomía }\end{array}$ & $\begin{array}{l}\text { Facilitación del crecimiento y sobrevivencia } \\
\text { de plántulas }\end{array}$ \\
\hline 2016 & Fernández & Colombia Forestal & Definición de objetivos y planificación \\
\hline 2016 & Manrique & $\begin{array}{l}\text { Bistua Revista de la Facultad } \\
\text { de Ciencias }\end{array}$ & Definición de objetivos y planificación \\
\hline 2016 & Gallego & Acta Biológica Colombiana & Establecimiento de plántulas a partir de semillas \\
\hline
\end{tabular}




\begin{tabular}{|c|c|c|c|}
\hline 2016 & Bare & New Forest & $\begin{array}{l}\text { Facilitación del crecimiento y sobrevivencia } \\
\text { de plántulas }\end{array}$ \\
\hline 2016 & Hernández & Tecnura & Resiliencia, sostenibilidad e integración con el paisaje \\
\hline 2016 & Posada & $\begin{array}{c}\text { Boletín Científico del Centro } \\
\text { de Museos }\end{array}$ & Definición de objetivos y planificación \\
\hline 2016 & Basham & Animal Conservation & Resiliencia, sostenibilidad e integración con el paisaje \\
\hline 2016 & $\begin{array}{l}\text { Gonzalez del } \\
\quad \text { Pliego }\end{array}$ & Biological Conservation & Resiliencia, sostenibilidad e integración con el paisaje \\
\hline 2016 & Valero & $\begin{array}{l}\text { Chemical and Biological Tecniques } \\
\text { in Agriculture }\end{array}$ & $\begin{array}{c}\text { Facilitación del crecimiento y sobrevivencia } \\
\text { de plántulas }\end{array}$ \\
\hline 2017 & Rojas & Caldasia & Resiliencia, sostenibilidad e integración con el paisaje \\
\hline 2017 & Solorza & Colombia Forestal & $\begin{array}{l}\text { Facilitación del crecimiento y sobrevivencia } \\
\text { de plántulas }\end{array}$ \\
\hline 2017 & Casallas & Acta Biológica Colombiana & Obtención de propágulos \\
\hline 2017 & Valois & Revista de Biología Tropical & Obtención de propágulos \\
\hline 2017 & Gutierrez & Revista de Biología Tropical & Establecimiento de plántulas a partir de semillas \\
\hline 2017 & Gutierrez & Revista de Biología Tropical & Obtención de propágulos \\
\hline 2017 & Rosselli & Acta Biológica Colombiana & Resiliencia, sostenibilidad e integración con el paisaje \\
\hline 2017 & Ocampo & Biota Colombiana & Definición de objetivos y planificación \\
\hline 2017 & Beltrán & Colombia Forestal & $\begin{array}{l}\text { Facilitación del crecimiento y sobrevivencia } \\
\text { de plántulas }\end{array}$ \\
\hline 2017 & López & Colombia Forestal & Establecimiento de plántulas a partir de semillas \\
\hline 2017 & Muñoz & Colombia Forestal & Obtención de propágulos \\
\hline 2017 & Clavijo & Producción Más Limpia & Definición de objetivos y planificación \\
\hline 2017 & Díaz & Biota Colombiana & Definición de objetivos y planificación \\
\hline 2017 & Díez & Colombia Forestal & Establecimiento de plántulas a partir de semillas \\
\hline 2017 & Thomas & Biodiversity and Conservation & Obtención de propágulos \\
\hline 2017 & González & Luna Azul & Definición de objetivos y planificación \\
\hline 2017 & Montealegre & Ornitología Colombiana & Resiliencia, sostenibilidad e integración con el paisaje \\
\hline 2017 & Galindo & Restoration Ecology & Obtención de propágulos \\
\hline 2017 & Galindo & Restoration Ecology & $\begin{array}{l}\text { Facilitación del crecimiento y sobrevivencia } \\
\text { de plántulas }\end{array}$ \\
\hline 2017 & Cubillos & $\begin{array}{l}\text { Revista Facultad Nacional } \\
\text { de Agronomía }\end{array}$ & Resiliencia, sostenibilidad e integración con el paisaje \\
\hline 2017 & Díez & Colombia Forestal & $\begin{array}{l}\text { Facilitación del crecimiento y sobrevivencia } \\
\text { de plántulas }\end{array}$ \\
\hline 2018 & Vásquez & Colombia Forestal & Definición de objetivos y planificación \\
\hline 2018 & Fuentes & Acta Biológica Colombiana & Definición de objetivos y planificación \\
\hline 2018 & Fuentes & Acta Biológica Colombiana & Definición de objetivos y planificación \\
\hline 2018 & Calderón & Caldasia & Establecimiento de plántulas a partir de semillas \\
\hline 2018 & Bocanegra & Tree Genetics \& Genomes & Obtención de propágulos \\
\hline 2018 & Tulande & Universitas Scientiarum & Resiliencia, sostenibilidad e integración con el paisaje \\
\hline 2018 & Castellanos & Land Degradation \& Development & Resiliencia, sostenibilidad e integración con el paisaje \\
\hline 2018 & Bocanegra & Biological Conservation & Obtención de propágulos \\
\hline 2018 & Jaramillo & Wetlands & Resiliencia, sostenibilidad e integración con el paisaje \\
\hline 2018 & Benavides & Journal of Soil and Water Conservation & Definición de objetivos y planificación \\
\hline 2018 & Vargas & Revista de Biología Tropical & Establecimiento de plántulas a partir de semillas \\
\hline 2018 & Vargas & Revista de Biología Tropical & Obtención de propágulos \\
\hline 2018 & Calderón & Caldasia & Obtención de propágulos \\
\hline 2018 & Peláez & Restoration Ecology & Resiliencia, sostenibilidad e integración con el paisaje \\
\hline 2018 & Domínguez & Sociobiology & Resiliencia, sostenibilidad e integración con el paisaje \\
\hline
\end{tabular}




\begin{tabular}{|c|c|c|c|}
\hline 2018 & Domínguez & Geoderma & Resiliencia, sostenibilidad e integración con el paisaje \\
\hline 2019 & Avella & Caldasia & Definición de objetivos y planificación \\
\hline 2019 & Suárez & Caldasia & Definición de objetivos y planificación \\
\hline 2019 & Suárez & Caldasia & Definición de objetivos y planificación \\
\hline 2019 & Bravo & $\begin{array}{l}\text { Revista de Investigación Agraria } \\
\text { y Ambiental }\end{array}$ & Definición de objetivos y planificación \\
\hline 2019 & Quiroga & $\begin{array}{c}\text { Boletín Científico del Centro } \\
\text { de Museos }\end{array}$ & Definición de objetivos y planificación \\
\hline 2019 & Torres & Caldasia & Definición de objetivos y planificación \\
\hline 2019 & Bocanegra & Caldasia & Obtención de propágulos \\
\hline 2019 & Díaz & Caldasia & $\begin{array}{c}\text { Facilitación del crecimiento y sobrevivencia } \\
\text { de plántulas }\end{array}$ \\
\hline 2019 & Gracia & Marine Pollution Bulletin & Definición de objetivos y planificación \\
\hline 2019 & Meyer & Carbon Balance and Management & Definición de objetivos y planificación \\
\hline 2019 & Domínguez & Ecological Indicators & Resiliencia, sostenibilidad e integración con el paisaje \\
\hline
\end{tabular}

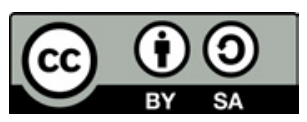

